\section{PLAN DE DESARROLLO COMUNAL: ¿EL INSTRUMENTO RECTOR DE LA GESTIÓN MUNICIPAL EN CHILE?}

Arturo Orellana², José Antonio Mena Valdés ${ }^{3}$ y

Martín Montes Marín ${ }^{4}$

\section{Resumen}

Este artículo cuestiona la idea de que el Plan de Desarrollo Comunal (PLADECO) es el "instrumento rector de la gestión comunal”, como plantea la legislación vigente. Para tal efecto realiza un análisis de contenido a partir de una muestra de 58 documentos PLADECO pertenecientes a comunas que son parte de las nueve áreas metropolitanas de Chile, tanto consolidadas como en proceso. En virtud de los objetivos que persigue este instrumento se establecen cuatro ejes de análisis: participación ciudadana, planificación, accesibilidad a la información y accountability, sobre los cuales se efectúa la revisión de los documentos, complementado este análisis con entrevistas a actores clave del mundo municipal, lo que permite conocer limitaciones y alcances del PLADECO. Se constata una

\section{MUNICIPAL DEVELOPMENT PLAN: THE GUIDING INSTRUMENT FOR MUNICIPAL MANAGEMENT IN CHILE?}

Arturo Orellana², José Antonio Mena Valdés ${ }^{3}$, Martín Montes Marín ${ }^{4}$

\section{Abstract}

This paper questions the belief that the Municipal Development Plan (PLADECO) serves - according to the current legislation - as the "guiding instrument for local municipal development". To contrast this idea, a content analysis was performed on a sample of 58 PLADECO documents from different Chilean urban municipalities. Four aspects were analyzed: citizen participation, strategic planning, information accessibility and accountability. In addition, interviews were conducted with key actors from various municipal systems in order to understand their views on the scope and limitations of PLADECO. Through this methodology, a high degree of heterogeneity was found in the levels of accomplishment of the analyzed 
alta heterogeneidad en el nivel de cumplimiento de los documentos revisados, destacando que los gobiernos locales se enfocan en cumplir con las exigencias de la legislación, mas no en utilizar este instrumento como la carta de navegación de la gestión municipal. Dentro de los ejes de análisis, las condiciones de accesibilidad resultan ser las mejor evaluadas, superando aspectos relativos a la planificación, participación y accountability. Por último, la voluntad política aparece como un factor fundamental en el desempeño del PLADECO.

\section{PALABRAS CLAVE: PLADECO, PLANIFICACIÓN ESTRATÉGICA， PARTICIPACIÓN CIUDADANA, ACCESIBILIDAD Y ACCOUNTABILITY.}

Recibido: 15-06-2015.

Aceptado: 16-05-2016.

1 Proyecto Anillos SOC1106: "Propuestas de rediseño institucional y reformulación de políticas públicas a partir del análisis de la incidencia de los gobiernos regionales y locales en la calidad de vida en las ciudades metropolitanas en Chile", financiado por la Comisión Nacional de Investigación Científica y Tecnológica (CONICYT).

2 Chile. Ingeniero Comercial y Licenciado en Economía (Universidad de Chile), Magíster en Desarrollo Urbano (PUC) y Doctor en Geografía Humana (Universidad de Barcelona). Profesor asociado, Instituto de Estudios Urbanos y Territoriales, Facultad de Arquitectura, Diseño y Estudios Urbanos de la PUC. Correo electrónico: amorella@uc.cl.

3 Chile. Sociólogo y Licenciado en Sociología de la Pontificia Universidad Católica de Chile. Correo electrónico: jamena@uc.cl.

4 Chile. Sociólogo y Licenciado en Sociología de la Pontificia Universidad Católica de Chile. Estudiante del Magíster en DesarroIlo Urbano del Instituto de Estudios Urbanos y Territoriales de la Pontificia Universidad Católica de Chile. Correo electrónico: m.montes@uc.cl. documents. The results show that local governments focus on following the requirements of the current legislation; however they rarely use them as a guiding tool for local administration. Accessibility conditions were the best assessed aspect, surpassing the areas of strategic planning, citizen participation and accountability. Finally, political will was found to be a fundamental factor in the performance of PLADECO.

\section{KEYWORDS: MUNICIPAL DEVELOPMENT PLAN; STRATEGIC PLANNING; CITIZEN PARTICIPATION; ACCESIBILITY; ACCOUNTABILITY.}

Received: 15-06-2015.

Accepted: 16-05-2016.

1 Anillos Project S0C1106: "Proposals for Institutional Redesign and Reformulation of Public Policies Based on the Analysis of the Influence of Regional and Local Governments on the Quality of Life in Chilean Metropolitan Cities", funded by CONICYT.

2 Chile. Commercial Engineer and BSc in Economy, University of Chile. MSc in Urban Development, Pontifical Catholic University, Chile. PhD in Human Geography, University of Barcelona. Professor, Institute of Urban and Territorial Studies at the Faculty of Architecture, Design and Urban Studies, Pontifical Catholic University, Chile.Email: amorella@ uc.cl.

3 Chile. Sociologist and BSc in Sociology, Pontifical Catholic University, Chile.Email: jamena@uc.cl.

4 Chile. Sociologist and BSc in Sociology, Pontifical Catholic University, Chile. Master's student in Urban Development, Institute of Urban and Territorial Studies at the Pontifical Catholic University, Chile.Email:m.montes@uc.cl. 


\section{Introducción y problema}

La expansión urbana y sus impactos en el desarrollo de los territorios es un fenómeno de escala planetaria $a^{5}$ que especialmente complejiza la tarea de planificar el desarrollo de las áreas metropolitanas. Según Fuentes y Orellana ${ }^{6}$, para el caso chileno la institucionalidad vigente no ha sido capaz de generar las condiciones de equilibrio socio-territorial en el desarrollo de las ciudades. Nuestras áreas metropolitanas muestran diferenciales de calidad de vida entre las comunas que las configuran, como los que se expresan a través del ICVU ${ }^{7}$, particularmente para el Área Metropolitana de Santiago (AMS).

Dentro de nuestra institucionalidad, los Instrumentos de Planificación Territorial (desde ahora IPT) se presentan como la gran vía para planificar y gestionar el desarrollo urbano de las ciudades, sin embargo, su eficacia e incidencia es discutible. Tal es así que la nueva Política Nacional de Desarrollo Urbano (PNDU) ${ }^{8}$ señala que actualmente "los instrumentos de planificación territorial tienen una cobertura sólo del $68 \%$ de las comunas,

\footnotetext{
5 Brenner, 2013.

6 Fuentes y Orellana, 2013.

7 Índice de Calidad de Vida Urbana (ICVU), desarrollado por el Instituto de Estudios Urbanos y Territoriales de la PUC y la Cámara Chilena de la Construcción.

tardan seis años en tramitarse y tienen pocas facultades para conducir el desarrollo urbano".

Existen tres IPT que son considerados como base para planificar y gestionar la comuna desde el municipio: el Plan Regulador Comunal (PRC), el Presupuesto Municipal Anual y el Plan de Desarrollo Comunal (PLADECO). No obstante, reconociendo la importancia de los dos primeros, este artículo centrará su análisis en los PLADECO, ya que de acuerdo a la legislación, este es el instrumento rector del desarrollo en la comuna?

Cabe señalar que aun cuando existen voces recientes que dan cuenta de la escasa incidencia que tiene este IPT en el desarrollo comunal ${ }^{10}$, se constata poca investigación que profundice en determinar por qué se da esta situación. Por ello es necesario revelar qué dificultades existen en su elaboración y ejecución, qué grado de participación ciudadana consideran, en qué medida operan como instrumento de planificación y si poseen alguna función efectiva como herramienta de rendición de cuentas de los gobiernos municipales frente a la ciudadanía. A partir de entrevistas y análisis de contenido de los documentos PLADECO, este trabajo pretende aportar a estos vacíos.

\footnotetext{
9 Ley 18.695, 2006.

10 Valdivieso, 2012.
} 


\section{Antecedentes teóricos}

\section{EL PLAN DE DESARROLLO COMUNAL}

La Ley $N^{0} 18.695^{11}$ señala que el Plan de Desarrollo Comunal, junto con el PRC y el Presupuesto Municipal Anual, corresponde a uno de los instrumentos mínimos para la gestión municipal y lo cataloga como el instrumento rector del desarrollo en la comuna. Junto a ello, la ley se refiere a los plazos de vigencia e indica la necesidad de tener en consideración la "participación ciudadana" y la coordinación con otros servicios públicos correspondientes en la elaboración y ejecución del plan.

Mientras la ley otorga lineamientos generales para la elaboración, ejecución y evaluación de los PLADECO, se dejan vacíos en la medida que no se hace un pronunciamiento explícito sobre el contenido y vinculación efectiva de los PLADECO con la gestión municipal, ni tampoco refiere a qué tipo de participación ciudadana, ni sobre la estructura que debe tener el instrumento, entre otras carencias relevantes ${ }^{12}$. Estas ambigüedades han generado que la elaboración de un PLADECO sea vista como una responsabilidad que un municipio debe cumplir, pero que no figuran como un verdadero insumo para la gestión comunal. En esta línea, Valdivieso ${ }^{13}$ se pregunta "¿Para qué sirve el PLADECO?” y añade: "Si uno hiciera una encuesta a personas con conocimiento de las dinámicas municipales en Chile, me atrevo a presagiar que la mayoría respondería 'para nada' o si fuera un poco más formal: 'para cumplir con la ley que dice que hay que tener un PLADECO vigente"'.

El debate sobre la injerencia que tienen los distintos IPT en Chile es una discusión que se ha estado llevando a cabo en el último tiempo y es en tal contexto en el que se sitúa esta investigación. Lo que se busca aquí es realizar un diagnóstico ya no basado en suposiciones o percepciones parciales desde el punto de vista de un solo municipio -tipo de visión donde se origina la mayor parte de las opiniones sobre este instrumento-, sino que un análisis empírico de mayor alcance que pueda dar cuenta en mayor medida hasta qué punto la realidad se corresponde con estas impresiones.

Desde esta situación, y reflexionando sobre el espíritu del PLADECO que se plasma en la ley mencionada, consideramos que éste debe servir de instrumento en tres dimensiones principales: planificación estratégica, participación ciudadana y rendición de cuentas (accountability). Sin embargo, a partir de esta última dimensión se incorporó una cuarta denominada accesibilidad, la cual tiene

\footnotetext{
11 Ley 18.695, 2006.

12 De la Maza, Campos, Vega y Gaete, 2008; Espinoza, 2010.

13 Valdivieso, 2012.
} 
que ver con el nivel de acceso y comprensión que tienen los ciudadanos frente al documento.

\section{PLANIFICACIÓN ESTRATÉGICA A NIVEL COMUNAL}

Durante las últimas décadas, la planificación estratégica ha pasado a formar parte esencial de las nuevas formas de gestión de los gobiernos en sus distintos niveles ${ }^{14}$. Así, a nivel local o comunal, la planificación estratégica consiste en trazar rumbos de gestión en el plano local, definiendo estratégicamente los objetivos y prioridades municipales ${ }^{15}$.

Es interesante señalar que "como instrumento de planificación, el PLADECO introdujo en el quehacer municipal una lógica de gestión sustentada en la construcción de un proyecto de comuna y una visión estratégica de las acciones necesarias para impulsar su desarrollo"16. En línea con una concepción estratégica de la gestión, este instrumento conforma un programa plurianual en las áreas de desarrollo económico, organización comunitaria, asistencia social, vivienda social, deporte y recreación, ordenamiento territorial, seguridad ciudadana, servicios públicos, medio ambiente, entre otras ${ }^{17}$. Sin embargo, en la práctica muchos municipios no realizan un seguimiento de lo

14 Sánchez, 2003.

15 Santana, 2012.

16 MIDEPLAN, 2006, s/p.

17 Santana, 2012. planificado en su respectivo PLADECO, transformando a este instrumento en una "carta de navegación ficticia"18. Al respecto, Santana ${ }^{19}$ sostiene que esta situación se debe principalmente a tres razones: i) la existencia de escasas atribuciones reales de los municipios para financiar proyectos; ii) la inexistencia de equipos profesionales y técnicos en los municipios, que estén capacitados para hacer el monitoreo de las iniciativas y para velar que se cumpla el PLADECO; y iii) la escasa relación del proceso de planificación con la ciudadanía, lo que dificulta que haya un proceso de control social. Estas falencias repercuten en que, para el caso de los PLADECO, la planificación estratégica falle en un nivel operacional.

En otra línea, según Jorquera y Pineda ${ }^{20}$, el PLADECO implica una planificación que trasciende el plazo de gobierno, generándose muchas veces un desinterés por parte de la autoridad política de utilizar el PLADECO y cumplir efectivamente con lo que en éste se estipula. A raíz de los distintos puntos de vista que hemos revisado, parece evidente que el PLADECO se enfrenta a una serie de desafíos pendientes que lo condicionan desde el corazón mismo del instrumento, vale decir, su capacidad de planificar la gestión municipal.

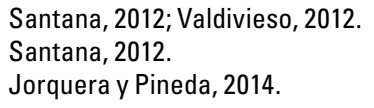




\section{EL PLADECO Y LA PARTICIPACIÓN CIUDADANA}

El PLADECO no sólo es un plan de desarrollo para la comuna, sino que también de la comuna; es decir que -por ley- éste debe involucrar a la comunidad en su elaboración por medio de un proceso de participación ciudadana. Según Caviglia ${ }^{21}$, la participación ciudadana apunta a dos grandes objetivos: el primero busca la sustitución o complementación de la democracia representativa y, el segundo busca influir en la administración de los recursos, intentando mejorar su funcionamiento en términos de eficacia y eficiencia. De este modo es factible asignar objetivos administrativos a la participación ciudadana ${ }^{22}$.

Siendo el PLADECO uno de los instrumentos claves de la gestión municipal, éste corresponde a una oportunidad para incorporar estos intereses a través de la participación ciudadana, un espacio que, estaría en deuda. Al respecto, Thayer y Elizalde ${ }^{23}$ nos dicen: "Esta mayor expectativa de representación que recae sobre los gobiernos locales exige, al menos en el caso de Chile, una ampliación de los espacios de participación, en función de convertir esa representación en una política efectiva". Específicamente en la elaboración del PLADECO, la participación se limita en la práctica a un nivel consultivo aplicada fundamentalmente en la fase

\footnotetext{
21 Caviglia, 1998.

22 Baño, 1998.

23 Thayer y Elizalde, 2011, p. 8.
}

de diagnóstico municipal, de tal manera que la ciudadanía no intervenga en etapas posteriores del proceso (implementación, control, fiscalización) ${ }^{24}$.

Remitiéndonos nuevamente a la ley, ésta no especifica qué se debe entender por "participación ciudadana" en lo que se refiere al PLADECO, por lo que procesos más o menos participativos y que pueden tener diferencias importantes en el nivel de vinculación que significan para la gestión, cumplen de igual forma con lo requerido en la ley, ya que ésta se satisface con la sola constatación de que se realizó participación ciudadana. Otro problema en este ámbito es que los procesos participativos asociados al PLADECO muchas veces no logran convocar a una ciudadanía diversa y representativa de los territorios ${ }^{25}$. Otra crítica frecuente es que en los procesos de participación, la ciudadanía es utilizada como un actor legitimador del instrumento de planificación ya diseñado "desde arriba", con el consiguiente alejamiento ciudadano respecto a la planificación de su territorio ${ }^{26}$.

\section{EL PLADECO COMO INSTRUMENTO DE ACCOUNTABILITY}

La participación ciudadana se liga indisolublemente al accountability., ya que en la medida que la población influye participativamente sobre la gestión

\footnotetext{
24 Montecinos, 2006.

25 Espinoza, 2010.

26 Jorquera y Pineda, 2014.
} 
de un gobierno, se vuelve probable que esta misma se interese por ver los resultados de aquellos proyectos en los cuales su opinión y trabajo estuvieron involucrados. En otras palabras, el aumento de la participación ciudadana es identificado como una instancia que facilita un mayor control social de la gestión pública por parte de la ciudadanía y la rendición de cuentas a ésta ${ }^{27}$.

Si bien el concepto de accountability generalmente se ha asociado con la transparencia de la información (ya sea de los proyectos en ejecución, del uso de los dineros públicos, etc.), su significado es mucho más profundo. Según San Martín ${ }^{28}$, accountability refiere a la capacidad de las instituciones políticas para hacer a sus gobernantes responsables de sus actos ${ }^{29}$. De esta forma, en la presente investigación, la dimensión de accountability se vincula a las herramientas que permitan llevar a cabo un control ciudadano de manera más directa (tales como un sistema de seguimiento o un sistema de sanciones a las autoridades por incumplimiento), distinguiendo de lo que tendrá directa relación con la transparencia de la información, lo cual será

27 Velásquez y González, 2003.

28 San Martín, 2008.

29 Crespo, 2001. denominado como accesibilidad, dimensión que considera el nivel de acceso y comprensión que se pone a disposición en los documentos analizados. En este sentido, para que exista una rendición de cuentas efectiva no basta con que la información esté disponible, sino que se hace necesario establecer distintos mecanismos institucionales que organicen la rendición de cuentas tanto de parte de quienes rinden como de quienes exigen cuentas ${ }^{30}$.

\section{OBJETIVOS Y METODOLOGÍA}

El presente artículo propone ahondar en las limitaciones y alcances que tiene actualmente el PLADECO como instrumento de planificación, participación y accountability en la gestión municipal. Para ello se realizó un análisis de contenido cualitativo de los documentos oficiales de los PLADECO de 58 de las 64 comunas $^{31}$ de las nueve áreas urbanas funcionales más importantes de

\footnotetext{
30 Sánchez, 2003.

31 Hubo sólo seis documentos que no pudieron ser analizados, ya que no se pudo tener acceso a la información en el momento que se realizó esta investigación: Independencia, Macul, Maipú, Santiago, Concepción y Talcahuano. Las nueve áreas funcionales de Chile comprenden: Iquique-Alto Hospicio, Antofagasta, La Serena-Coquimbo, Gran Valparaíso, Gran Santiago, RancaguaMachalí, Gran Concepción, Temuco-Padre Las Casas y Puerto Montt-Puerto Varas.
} 
Chile, según los criterios de la OCDE-SUBDERE ${ }^{32}$, considerando áreas metropolitanas consolidadas y otras en proceso de consolidación. Con esta metodología se verificó la presencia o ausencia de determinados temas por medio de una codificación sistemática de los textos analizados ${ }^{33}$. Cabe precisar que los documentos analizados representan el $16,7 \%$ del total de municipios del país (58/346) y el 90,6\% del total de municipios pertenecientes a áreas urbanas funcionales (58/64).

En segundo lugar se buscó conocer las distintas experiencias a nivel municipal en torno a los procesos de elaboración e implementación del PLADECO. Con tal propósito se realizaron 18 entrevistas semi-estructuradas a funcionarios municipales que estuvieron vinculados de forma

32 Según OCDE-SUBDERE (2013), un área urbana funcional es aquella que se configura a partir de dos o más comunas con un núcleo central de más de 50.000 habitantes que configuran relaciones de flujo por motivos laborales correspondientes a más del $15 \%$ de la población.

33 Para el análisis de los textos se elaboró un cuestionario de 33 preguntas dicotómicas (Sí/No) distribuidas en torno a 4 temas: participación, planificación, accesibilidad y accountability. Junto a esto, para asegurar confiabilidad y validez en el estudio, se definieron criterios de respuesta para cada pregunta, los que fueron aplicados de forma rígida sobre los contenidos. cercana con el proceso de elaboración e implementación del PLADECO de su comuna ${ }^{34}$.

Algunas limitaciones metodológicas que es necesario tener en consideración es que por medio del análisis de documentos sólo se verificó la ausencia o presencia de distintos ítems, sin saber si existe un correlato de éstos con la realidad. De esta manera, los resultados presentados se remiten únicamente a lo que los documentos declaran, por lo tanto, no es posible realizar juicios sustantivos sobre éstos. Junto a esto, es relevante considerar también que el análisis de los documentos logra capturar una "foto del pasado", más no del presente ya que si bien se revisaron los PLADECO más actualizados de cada municipio, no todos estaban vigentes, siendo algunos de ellos de larga data de uso. En este sentido es posible que los instrumentos que se estén elaborando en el último tiempo tengan características distintas a los que se muestran en los resultados de este estudio.

34 Se seleccionaron dos municipios por cada una de las áreas metropolitanas más grandes (Gran Santiago, Gran Valparaíso y Gran Concepción). Los 6 municipios con los que se trabajó fueron escogidos buscando la mayor heterogeneidad posible según antigüedad del PLADECO, tamaño de población y posición del ranking ICVU 2014. Los municipios seleccionados fueron Providencia, Lo Prado, Viña del Mar, Villa Alemana, San Pedro de la Paz y Coronel. 


\section{Análisis por dimensión de los PLADECO}

Los resultados que se presentan a continuación corresponden a una síntesis de los resultados cuantificables obtenidos a partir del análisis de contenido llevado a cabo sobre cada uno de los documentos. En base a esto se pudo determinar qué dimensiones están cubiertas en un panorama general y cuáles lo están menos. En esta línea se propone una tipología que permite aunar documentos que tienen ciertas similitudes en el cumplimiento de los aspectos evaluados. En la segunda sección se expone con mayor detalle un análisis de cada una de las dimensiones estudiadas, incorporando ideas obtenidas de las entrevistas efectuadas al personal de seis municipios, que sean pertinentes para guiar una reflexión.

\section{UNA TIPOLOGÍA PARA LOS PLADECO}

Los documentos del PLADECO fueron evaluados según la constatación de determinados contenidos relativos a cuatro dimensiones: planificación estratégica, participación ciudadana, accesibilidad y accountability. A partir de los resultados del análisis se construyeron cuatro índices para medir respectivamente el nivel de cumplimiento de cada PLADECO en las áreas evaluadas. Los índices son comparables entre sí y asumen un rango de valores entre 0 y 1 , refiriendo el porcentaje de cumplimiento de cada PLADECO respecto del total de ítems analizados en cada dimensión. Además, los cuatro índices temáticos fueron promediados dando origen a un indicador que da cuenta del cumplimiento general de cada PLADECO para los contenidos evaluados (Índice General de Cumplimiento, IGC). No obstante se reconoce su condición de indicador global de carácter simple, permite establecer en líneas generales una evaluación comparada del nivel de logro entre PLADECOS, desde la percepción de los actores municipales vinculados.

A partir de la figura 1 es relevante rescatar que a medida que el porcentaje de cumplimiento de cada PLADECO en las distintas dimensiones analizadas se hace menor (disminuyendo así el IGC), aumenta la heterogeneidad que existe en el nivel de cumplimiento de las distintas dimensiones analizadas.

Junto a esto se construyó una tipología para los PLADECO, la cual permite categorizar y dar cuenta del grado de heterogeneidad que éstos tienen respecto de los contenidos que se incluyen/excluyen en las dimensiones estudiadas. La tipología propuesta se determinó en función de cuántos índices por sobre el promedio del total obtuvo cada documento ${ }^{35}$. Los PLADECO tipo A -con el mejor rendimiento conforme a lo evaluado- corresponden

35 Se consideran valores mayores ( $>$ ), no mayor o igual $(\geq)$. 


\section{FIGURA 1. COMPORTAMIENTO COMPARADO DE ÍNDICES, ORDENADOS SEGÚN IGC.}

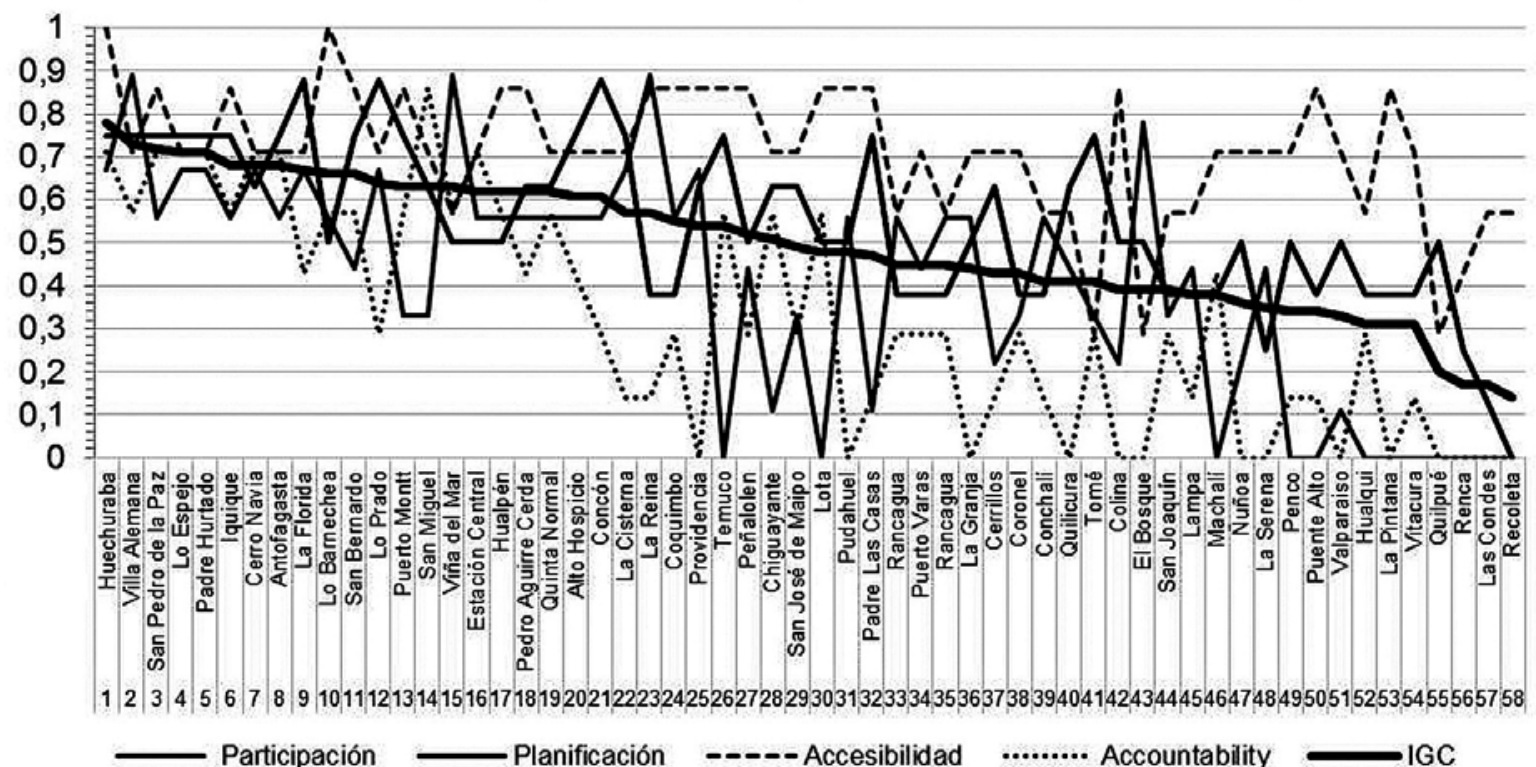

Fuente: elaboración propia.

a todos aquellos documentos que tengan índices sobre el promedio general en las cuatro dimensiones analizadas; los tipo B corresponden a los documentos que obtuvieron un índice mayor que el promedio en 3 de 4 dimensiones; los tipo C corresponden a los documentos que obtuvieron un índice mayor que el promedio en 2 de 4 dimensiones; los tipo D corresponden a los documentos que obtuvieron un índice mayor que el promedio solamente en 1 de 4 dimensiones; por último, los PLADECO tipo E corresponden a aquellos documentos que obtuvieron un índice bajo el promedio en todas las dimensiones evaluadas.

La figura 2 representa una síntesis agregada de los resultados obtenidos por cada tipo de PLADECO en las 4 dimensiones estudiadas, en donde a medida que el cuadrante es mayor, hay un mayor nivel de cumplimiento de los ítems analizados. A continuación se detallan los 5 tipos de PLADECO definidos. 


\section{FIGURA 2. COBERTURA POR DIMENSIONES SEGÚN TIPOLOGÍA PLADECO.}

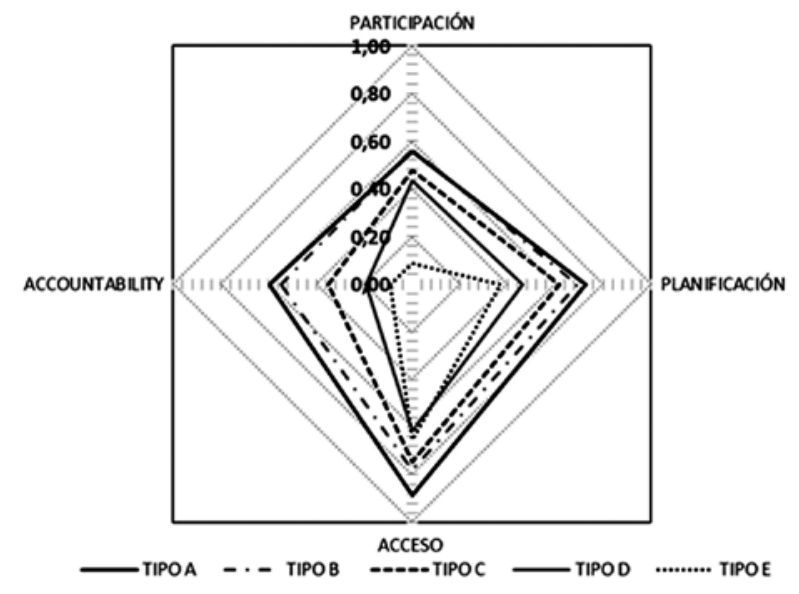

Fuente: elaboración propia.

\section{A) PLADECO TIPO A}

Este grupo abarca a los PLADECO que tuvieron un índice sobre el promedio en todas las dimensiones evaluadas. De los 58 casos estudiados hay 5 documentos tipo A (Huechuraba, Iquique, Pedro Aguirre Cerda, San Bernardo y San Pedro de la Paz). En lo relativo a la dimensión de planificación, los PLADECO tipo A cumplieron en promedio con el 73\% de la pauta de análisis de contenido (índi$\mathrm{ce}=0.73$ ). En participación ciudadana promediaron 0.56 , en accesibilidad tuvieron una media de 0.89 y en accountability se obtuvo un promedio de 0.6 , número muy similar al obtenido para participación ciudadana. Promediando todas las categorías, los PLADECO tipo A obtuvieron un IGC de 0.69.

TABLA 1. RESULTADOS DE LOS PLADECO TIPO A.

\begin{tabular}{lccccc} 
COMUNA & PARTICIPACIÓN & PLANIFICACIÓN & ACCESIBILIDAD & ACCOUNTABILITY & IGC \\
\hline Huechuraba & 0.67 & 0.75 & 1.00 & 0.71 & 0.78 \\
San Pedro de la Paz & 0.56 & 0.75 & 0.86 & 0.71 & 0.72 \\
Iquique & 0.56 & 0.75 & 0.86 & 0.57 & 0.68 \\
San Bernardo & 0.44 & 0.75 & 0.86 & 0.57 & 0.66 \\
\hline Pedro Aguirre Cerda & 0.56 & 0.63 & 0.86 & 0.43 & 0.62 \\
Promedio Tipo A & 0.56 & 0.73 & 0.89 & 0.60 & 0.69 \\
Promedio total & 0.4 & 0.55 & 0.71 & 0.32 & 0.5
\end{tabular}

Fuente: elaboración propia, 2015. 


\section{B) PLADECO TIPO B}

Este grupo abarca a los PLADECO que obtuvieron un índice sobre el promedio en 3 de las 4 dimensiones evaluadas. De los 58 casos estudiados hay 13 documentos tipo B. En lo relativo a la dimensión de planificación, los PLADECO tipo B cumplieron en promedio con el 69\% de la pauta de análisis de contenido. En participación ciudadana promediaron un índice de 0.57 , en accesibilidad tuvieron un índice medio de 0.78 y en accountability se obtuvo un promedio de 0.55 , también muy similar al obtenido para participación ciudadana (al igual que en el tipo A). Promediando todas las categorías, los PLADECO tipo B obtuvieron un IGC de 0.65.

TABLA 2. RESULTADOS DE LOS PLADECO TIPO B.

\begin{tabular}{lcccccc}
\hline COMUNA & PARTICIPACIÓN & PLANIFICACIÓN & ACCESIBILIDAD & ACCOUNTABILITY & IGC \\
\hline Villa Alemana & 0.89 & 0.75 & 0.71 & 0.57 & 0.73 \\
\hline Lo Espejo & 0.67 & 0.75 & 0.71 & 0.71 & 0.71 \\
\hline Padre Hurtado & 0.67 & 0.75 & 0.71 & 0.71 & 0.71 \\
\hline Antofagasta & 0.56 & 0.75 & 0.71 & 0.71 & 0.68 \\
\hline Cerro Navia & 0.67 & 0.63 & 0.71 & 0.43 & 0.68 \\
\hline La Florida & 0.67 & 0.88 & 0.71 & 0.57 & 0.67 \\
Lo Barnechea & 0.56 & 0.50 & 1.00 & 0.57 & 0.66 \\
\hline Puerto Montt & 0.33 & 0.75 & 0.86 & 0.57 & 0.63 \\
\hline Hualpén & 0.56 & 0.50 & 0.71 & 0.57 & 0.62 \\
\hline Cuinta Normal & 0.56 & 0.63 & 0.71 & 0.43 & 0.62 \\
\hline Alto Hospicio & 0.56 & 0.75 & 0.86 & 0.00 & 0.61 \\
\hline Providencia & 0.67 & 0.63 & 0.86 & 0.57 & 0.54 \\
\hline Temuco & 0.00 & 0.75 & 0.78 & 0.55 & 0.54 \\
\hline Promedio Tipo B & 0.57 & 0.69 & 0.71 & 0.32 & 0.65
\end{tabular}

Fuente: elaboración propia, 2015. 


\section{C) PLADECO TIPO C}

Este grupo abarca a los PLADECO que obtuvieron un índice sobre el promedio en 2 de las 4 dimensiones evaluadas. De los 58 casos estudiados hay 14 documentos tipo C. En lo relativo a la dimensión de planificación, los PLADECO tipo C cumplieron en promedio con el $60 \%$ de la pauta de análisis de contenido. En participación ciudadana promediaron un índice de 0.49 , en accesibilidad tuvieron un índice medio de 0.75 y en accountability se obtuvo un índice de 0.35 . Promediando todas las categorías, los PLADECO tipo C obtuvieron un IGC de 0.55 .

\section{TABLA 3. RESULTADOS DE LOS PLADECO TIPO C.}

\begin{tabular}{|c|c|c|c|c|c|}
\hline COMUNA & PARTICIPACIÓN & PLANIFICACIÓN & ACCESIBILIDAD & ACCOUNTABILITY & IGC \\
\hline Lo Prado & 0.67 & 0.88 & 0.71 & 0.29 & 0.64 \\
\hline San Miguel & 0.33 & 0.63 & 0.71 & 0.86 & 0.63 \\
\hline Viña del Mar & 0.89 & 0.50 & 0.57 & 0.57 & 0.63 \\
\hline Estación Central & 0.56 & 0.50 & 0.71 & 0.71 & 0.62 \\
\hline Concón & 0.56 & 0.88 & 0.71 & 0.29 & 0.61 \\
\hline La Reina & 0.89 & 0.38 & 0.86 & 0.14 & 0.57 \\
\hline La Cisterna & 0.67 & 0.75 & 0.71 & 0.14 & 0.57 \\
\hline Coquimbo & 0.56 & 0.38 & 0.86 & 0.29 & 0.55 \\
\hline Peñalolén & 0.44 & 0.50 & 0.86 & 0.29 & 0.52 \\
\hline Chiguayante & 0.11 & 0.63 & 0.71 & 0.57 & 0.51 \\
\hline Lota & 0.00 & 0.50 & 0.86 & 0.57 & 0.48 \\
\hline Pudahuel & 0.56 & 0.50 & 0.86 & 0.00 & 0.48 \\
\hline Padre Las Casas & 0.11 & 0.75 & 0.86 & 0.14 & 0.47 \\
\hline Quilicura & 0.44 & 0.63 & 0.57 & 0.00 & 0.41 \\
\hline Promedio Tipo C & 0.49 & 0.60 & 0.75 & 0.35 & 0.55 \\
\hline Promedio total & 0.4 & 0.55 & 0.71 & 0.32 & 0.5 \\
\hline
\end{tabular}

Fuente: elaboración propia, 2015. 


\section{D) PLADECO TIPO D}

Este grupo abarca a los PLADECO que obtuvieron un índice sobre el promedio en sólo 1 de las 4 dimensiones evaluadas. De los 58 casos estudiados hay 13 documentos tipo D. En lo relativo a la dimensión de planificación, los PLADECO tipo D cumplieron en promedio con el $46 \%$ de la pauta de análisis de contenido. En participación ciudadana promediaron un índice de 0.38 , en accesibilidad tuvieron un índice medio de 0.64 y en accountability se obtuvo un índice de 0.15 . Promediando todas las categorías, los PLADECO tipo D obtuvieron un IGC de 0.41 .

\section{TABLA 4. RESULTADOS DE LOS PLADECO TIPO D.}

\begin{tabular}{lccccc}
\hline COMUNA & PARTICIPACIÓN & PLANIFICACIÓN & ACCESIBILIDAD & ACCOUNTABILITY & IGC \\
\hline San José de Maipo & 0.33 & 0.63 & 0.71 & 0.29 & 0.49 \\
\hline Puerto Varas & 0.44 & 0.38 & 0.71 & 0.29 & 0.45 \\
Rancagua & 0.56 & 0.38 & 0.57 & 0.29 & 0.45 \\
La Granja & 0.56 & 0.50 & 0.71 & 0.00 & 0.44 \\
Cerrillos & 0.22 & 0.63 & 0.71 & 0.14 & 0.43 \\
\hline Tomé & 0.33 & 0.75 & 0.29 & 0.29 & 0.41 \\
Conchalí & 0.56 & 0.38 & 0.57 & 0.14 & 0.41 \\
El Bosque & 0.78 & 0.50 & 0.29 & 0.00 & 0.39 \\
Colina & 0.22 & 0.50 & 0.86 & 0.00 & 0.39 \\
Machalí & 0.00 & 0.38 & 0.71 & 0.43 & 0.38 \\
Lampa & 0.44 & 0.38 & 0.57 & 0.14 & 0.38 \\
La Serena & 0.44 & 0.25 & 0.71 & 0.00 & 0.35 \\
La Pintana & 0.00 & 0.38 & 0.86 & 0.00 & 0.31 \\
\hline Promedio Tipo D & 0.38 & 0.46 & 0.64 & 0.71 & 0.15
\end{tabular}

Fuente: elaboración propia, 2015. 


\section{E) PLADECO TIPO E}

Este grupo abarca a los PLADECO que no obtuvieron ninguno de sus índices por sobre el promedio general las dimensiones evaluadas. De los 58 casos estudiados hay 12 documentos tipo E. En lo relativo a la dimensión de planificación, los PLADECO tipo E cumplieron en promedio con el
36\% de la pauta de análisis de contenido. En participación ciudadana promediaron un índice de 0.08 , en accesibilidad tuvieron un índice medio de 0.62 y en accountability se obtuvo un índice de 0.11 (valor similar al de participación, tal como en los tipo A y B). Promediando todas las categorías, los PLADECO tipo E obtuvieron un IGC de 0.29.

\section{TABLA 5. RESULTADOS DE LOS PLADECO TIPO E.}

\begin{tabular}{lccccc}
\hline COMUNA & PARTICIPACIÓN & PLANIFICACIÓN & ACCESIBILIDAD & ACCOUNTABILITY & IGC \\
\hline Coronel & 0.33 & 0.38 & 0.71 & 0.29 & 0.43 \\
San Joaquín & 0.33 & 0.38 & 0.57 & 0.29 & 0.39 \\
\hline Nuñoa & 0.22 & 0.50 & 0.71 & 0.00 & 0.36 \\
\hline Penco & 0.00 & 0.50 & 0.71 & 0.14 & 0.34 \\
\hline Puente Alto & 0.00 & 0.38 & 0.86 & 0.14 & 0.34 \\
Valparaíso & 0.11 & 0.50 & 0.71 & 0.00 & 0.33 \\
\hline Hualqui & 0.00 & 0.38 & 0.57 & 0.29 & 0.31 \\
Vitacura & 0.00 & 0.38 & 0.71 & 0.14 & 0.31 \\
Quilpué & 0.00 & 0.50 & 0.29 & 0.00 & 0.20 \\
Las Condes & 0.00 & 0.13 & 0.57 & 0.00 & 0.17 \\
Renca & 0.00 & 0.25 & 0.43 & 0.00 & 0.17 \\
Recoleta & 0.00 & 0.00 & 0.57 & 0.00 & 0.14 \\
\hline Promedio Tipo E & 0.08 & 0.36 & 0.62 & 0.11 & 0.29 \\
Promedio total & 0.4 & 0.55 & 0.71 & 0.32 & 0.5
\end{tabular}

Fuente: elaboración propia, 2015. 
TABLA 6. VALOR PROMEDIO DE ÍNDICES POR DIMENSIÓN.

\begin{tabular}{ccccc} 
Planificación & Participación & Accountability & Accesibilidad & IGC \\
\hline 0.55 & 0.4 & 0.32 & 0.71 & 0.5
\end{tabular}

Fuente: elaboración propia, 2015.

Ya habiendo caracterizado los distintos tipos de PLADECO, en términos generales se observa que la dimensión accesibilidad es la mejor abarcada por los documentos analizados, presentando pocas variaciones en su completitud de contenidos entre documentos que pueden diferir mucho unos de otros respecto de sus evaluaciones en las dimensiones restantes. De este modo, se puede concluir que existe poca heterogeneidad "externa" o entre-documentos respecto de las posibilidades que éstos ofrecen para la accesibilidad del documento. Por otro lado, es interesante notar que a nivel agregado parece haber una correlación entre el índice de participación y el de accountability, de tal manera que aquellos documentos con buen índice de participación tienden a presentar mejores resultados en rendición de cuentas y viceversa.

\section{RESULTADOS POR DIMENSIÓN}

A continuación se analizan los resultados obtenidos según cada una de las dimensiones analizadas. Los valores promedios se muestran en la tabla 6 .

\section{Planificación estratégica}

Proponer una planificación estratégica para la gestión de municipios constituye quizás el primer objetivo del PLADECO. El análisis realizado da cuenta de que existe una heterogeneidad importante en relación al nivel de rigor y profundidad del sentido planificador de los documentos revisados.

Para evaluar en qué medida los PLADECO cumplen con su rol de planificación se seleccionaron 8 ítems y a partir de éstos se calculó un índice de planificación, el cual refleja en qué medida el documento aborda la planificación estratégica y operativa de la gestión municipal. El promedio obtenido de la muestra es 0.55 , lo que quiere decir que en promedio los PLADECO cumplieron con el 55\% de los contenidos exigidos por la pauta de evaluación en este aspecto, siendo el segundo ítem mejor evaluado (después de accesibilidad y comprensión: 71\%). Las mayores puntuaciones las obtuvieron los documentos de La Florida, Lo Prado y Concón (0.88), y la puntuación más baja corresponde a Recoleta (0). 
Al profundizar en este aspecto se destaca que los PLADECO revisados -en cuanto a planificación se refiere- por sobre cualquier otro de los ítems estudiados, se centran principalmente en incluir un diagnóstico comunal ${ }^{36}$, así como en el planteamiento de objetivos y en incorporar iniciativas concretas de acción que respondan a los objetivos propuestos. Esto quiere decir que la gran mayoría de los documentos revisados cuenta con estos elementos (alrededor del 90\% posee diagnóstico, objetivos e iniciativas concretas de acción), lo cual nos habla que actualmente pareciera haber consenso de que estos elementos forman parte de los mínimos necesarios para una planificación estratégica adecuada (figura 3).

Sin embargo, al profundizar en el tema de la planificación se observan resultados muy distintos a los recién mencionados. En este sentido, al analizar ítems como la consideración de indicadores ${ }^{37}$, la coordinación con otros niveles de gestión ${ }^{38}$, la incorporación de

36 Se consideró si se hizo una descripción general de los distintos sectores y aspectos de desarrollo de la comuna, generando así una situación inicial sobre la cual es posible plantear objetivos.

37 Se evaluó la presencia de indicadores de desempeño -tanto cualitativos como cuantitativos- que permitan monitorear el cumplimiento de los objetivos e iniciativas planificadas.

38 Se evaluó si la planificación estratégica comunal expuesta se encuentra articulada con políticas, instrumentos y estrategias propios de otros niveles de gestión tales como el intercomunal y/o regional (ejemplo: Estrategia de Desarrollo Regional).

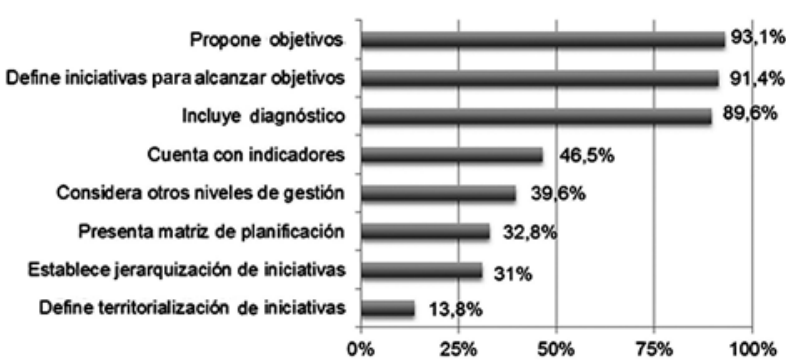

Fuente: elaboración propia.

una matriz de planificación ${ }^{39}$, la jerarquización de objetivos e iniciativas ${ }^{40}$ y la territorialización de las iniciativas planteadas ${ }^{41}$, la cobertura que se tiene es muchísimo menor. En esta línea, todos los ítems mencionados anteriormente son cubiertos por menos de la mitad de los documentos revisados, siendo el más cubierto de éstos la incorporación

39 Como una forma básica de sintetizar y ordenar los elementos centrales de la planificación se revisó que el documento presentara un cuadro o matriz que al menos contuviera objetivos, iniciativas e indicadores alineados entre sí.

40 Se verificó si se planteaba que el desarrollo de determinados objetivos y lineamientos constituiría una prioridad para la gestión, en relación a otros objetivos y lineamientos considerados como de menor prioridad.

41 Se evaluó que en aquellas iniciativas en que fuera pertinente se indique la localización de ésta en el territorio, o bien que se presenten planes de acción específicos para cada territorio de la comuna. 
de indicadores de desempeño (46,5\%) y el menos cubierto la territorialización de las iniciativas planteadas (13,8\%).

Los resultados recién expuestos dan cuenta de que -por lo general- los PLADECO se quedan en realizar diagnósticos temáticos y proponer objetivos e iniciativas, pero no profundizan en aspectos más concretos de la planificación, que es donde se desarrollan las estrategias para saber cómo lograr dichos objetivos. Las distintas experiencias municipales estudiadas a través de las entrevistas nos indican que ésta podría ser una razón importante de por qué los PLADECO tienen dificultades para traducirse en proyectos concretos que impacten en el territorio.

Según los entrevistados, uno de los principales obstaculizadores para avanzar en materia de planificación se debe a que existe una fuerte tensión entre la planificación y la contingencia, es decir, entre lo que puede ser planificado con una visión a largo plazo y los sucesos imprevistos que surgen en instituciones dinámicas como los municipios. Este trade-off entre planificación y contingencia en parte se debe a que las instituciones municipales son sumamente dinámicas. Además de ser instituciones dinámicas porque ocurren situaciones inesperadas, también lo son porque los recursos son escasos, lo cual obliga a que los municipios tengan sus puertas abiertas para poder aprovechar cualquier oportunidad que se les presenta. En este sentido, los gobiernos locales deben saber aprovechar los recursos que inesperadamente ofrecen los privados o el gobierno central. Por otro lado, la gestión municipal es dinámica también por una razón política. Algunos entrevistados señalaron que las autoridades políticas están más interesadas en llevar adelante iniciativas populares que les permitan ganar futuras elecciones, y en este sentido, la planificación a largo plazo coarta en alguna medida una flexibilidad que es valiosa para manejar la contingencia optimizando los réditos políticos.

Esta tensión entre planificación y contingencia exige preguntarse hasta qué nivel es necesario planificar y permite darle importancia al hecho de que, como dice el manual de elaboración del PLADECO $^{42}$, este instrumento debe ser flexible. A raíz de lo mencionado podemos concluir que, a diferencia de las empresas privadas, los gobiernos locales poseen muchas particularidades que les exigen una mayor flexibilización de sus objetivos, por lo tanto, se presenta el gran desafío de buscar la forma de compatibilizar la visión a largo plazo con las nuevas necesidades que van surgiendo día a día. Según la experiencia de los municipios con los que se trabajó, una posible respuesta para solucionar este problema es la actualización y uso constante del instrumento. En este sentido, uno de los entrevistados opina que es necesario 
ir "revisando año a año los planes territoriales, y trabajando constantemente en actualizar las prioridades con la comunidad, lo cual le da flexibilidad al PLADECO para que no sea un instrumento rígido que quede tirado por ahí. Tú no actualizas el PLADECO cada 4 años, le tienes que estar dando vida permanentemente." (asesor de la administración municipal de Providencia)

Por otro lado, las experiencias municipales revisadas dieron a conocer que un gran facilitador para la planificación es la coordinación entre los proyectos municipales con los regionales o de mayor escala. En dicha línea se señaló que si hubiera una coordinación real de los gobiernos locales con los regionales, habría también una mayor coordinación en el desarrollo de las comunas vecinas dentro de la región, lo cual favorecería a la coordinación intercomunal, cuestión que en el contexto de ciudades metropolitanas es sumamente necesaria. Esta coordinación también se vuelve necesaria por un hecho práctico; dado que la gran mayoría de los municipios depende financieramente de las entidades centrales, para llevar a cabo sus proyectos deben coordinarse con las líneas de desarrollo que éste propone. Sin embargo, según los entrevistados, esto a su vez pasa a ser un problema ya que por la necesidad de obtener los recursos se le da prioridad a los proyectos del gobierno central por sobre los locales. Esta situación afecta a la planificación de manera directa pues si no tengo certeza de la cantidad de recursos que voy a tener, no sé hasta qué punto puedo planificar, ya que puede que no tenga cómo llevar a cabo los programas y proyectos previamente planificados. Al respecto, uno de los entrevistados señala: "Dependemos del fondo común municipal. Como en un 60\% o 70\% (...) hay que buscar recursos en el gobierno regional a través del FNDR, del FRI, del PMU y toda esa cosa. Entonces, a pesar de que tú planifiques, después te encuentras con la dificultad de que no puedes concretar por falta de recursos, esa es la mayor dificultad" (asesor de la SECPLA, Coronel). De este modo, si bien es necesario que la planificación se realice de manera coordinada con las entidades superiores, a la vez se debe ser cuidadoso con abandonar la planificación local que permite responder a problemáticas específicas que se levantan en cada localidad, las cuales las unidades superiores no son capaces de visibilizar.

Junto a esto, el hecho de no tener los recursos necesarios para desarrollar los proyectos planificados lleva a no poder contratar suficiente personal calificado, el cual es el que tiene el conocimiento técnico para elaborar estrategias que permitan llevar a cabo los objetivos planteados y desarrollar proyectos para postular a los fondos de financiamiento externos, por lo tanto se va generando un ciclo del que cuesta salir. Esto se relaciona también con otro obstaculizador que, según la SECPLA de Viña del Mar, afecta a la planificación; nos referimos a que el PLADECO no tiene correspondencia con la realidad organizacional de la municipalidad. Este 
tipo de situaciones nos permiten conocer qué factores inciden en que por ejemplo más de la mitad de los documentos revisados no presenten indicadores de desempeño que permitan monitorear la gestión.

\section{Participación ciudadana}

La Ley No 18.695 señala que "en la elaboración y ejecución del plan comunal de desarrollo, tanto el alcalde como el Concejo deberán tener en cuenta la participación ciudadana"³. Sin embargo, más allá de formalizar el hecho de que legalmente los municipios tienen la obligación de considerar algún tipo de proceso participativo en este contexto, la ley citada no expresa mayor especificidad en términos de qué se entiende por participación ni cuáles son los mecanismos legítimos para que ésta sea tal. En este sentido y en ausencia de un estándar normado, es esperable encontrar un nivel importante de heterogeneidad respecto de las características particulares de los distintos procesos de participación ciudadana que se llevaron a cabo para elaborar los PLADECO observados en este estudio. A continuación se presentan los resultados descriptivos obtenidos en lo relativo a participación ciudadana.

El índice de participación ciudadana refleja en qué medida se constata y describe en el PLADECO los

43 Ley $18.695,2006$.

192 revista invi 31(87) : 173-200, agosto de 2016 distintos ámbitos de la realización de un proceso de participación ciudadana. El promedio obtenido de la muestra es 0.40 , lo que quiere decir que en promedio los PLADECO cumplieron con el 40\% de los contenidos exigidos en la pauta de evaluación en este aspecto. Las mayores puntuaciones las obtuvieron los documentos de Villa Alemana y Viña del Mar $(0,89)$ y la puntuación más baja corresponde a Hualqui, La Pintana, Las Condes, Lota, Machalí, Penco, Quilpué, Recoleta, Renca, Temuco y Vitacura (0).

En primer lugar podemos señalar que varios documentos explicitan que hubo participación ciudadana en su elaboración (77,6\%). No obstante, hay que considerar que por un lado la ley exige que se cuente con un proceso participativo, y también que actualmente la participación ciudadana ha pasado a ser un elemento que se ha puesto de moda y que suele estar muy presente en los discursos, pero éstos por lo general se quedan en retórica o, como dice Arnstein ${ }^{44}$, en un rito vacío. El gran desafío entonces estaría puesto en dilucidar de qué manera fue aplicada la participación ciudadana a la hora de elaborar y ejecutar el PLADECO.

De este modo, una vez que profundizamos en el ámbito de la participación nos encontramos con que en general los PLADECO no se refieren a cómo fueron los procesos de participación. Los únicos
44 Arnstein, 1969.

ARTÍCULO: Plan de desarrollo comunal: ¿El instrumento rector de la gestión municipal en Chile?/Arturo Orellana, José Antonio Mena Valdés, Martín Montes Marín 
ítems que fueron cubiertos por al menos más de la mitad de la muestra corresponden a que el PLADECO señala los métodos utilizados ${ }^{45}(67,3 \%)$ y que expone resultados del proceso participativo (53,5\%). Por su parte, la identificación de cuándo hubo participación (en qué etapas) y la consideración de la participación en la planificación ${ }^{46}$ fueron ítems cubiertos por alrededor de un $40 \%$ de los documentos revisados. Para todos los demás ítems evaluados, tales como explicitación del nivel de vinculación ${ }^{47}$, identificación de las organizaciones sociales que participaron ${ }^{48}$, caracterización de los participantes ${ }^{49}$ y explicitación de si hubo representación territorial ${ }^{50}$, la cobertura fue menor al 27\% (figura 4).

45 Hace referencia a las técnicas concretas que se emplearon para llevar a cabo la participación ciudadana.

46 Cuando se indica de manera explícita que los puntos de vista de la comunidad se incorporaron en los objetivos y/o proyectos planteados.

47 Se consideraron distintos niveles de vinculación participativa: informativa, consultiva, decisoria, co-gestión, control social, o equivalentes.

48 Es decir, si señala la naturaleza de las organizaciones sociales que participaron (centros de madres, clubes deportivos, grupos ambientalistas, centros de alumnos, etc.), no es necesario especificar el nombre de la organización.

49 Se consideraron dos criterios: (i) Cuando se hace una descripción de las características demográficas de quienes participaron y/o (ii) Cuando se mencionan de manera explícita los grupos de interés que participaron.

50 Hace alusión a la procedencia de quienes participaron, abordando una división clara del territorio (ya sea en unidades vecinales o barrios) buscando así que la comunidad sea representada por gente de todos sus sectores.

\section{FIGURA 4. PARTICIPACIÓN CIUDADANA.}

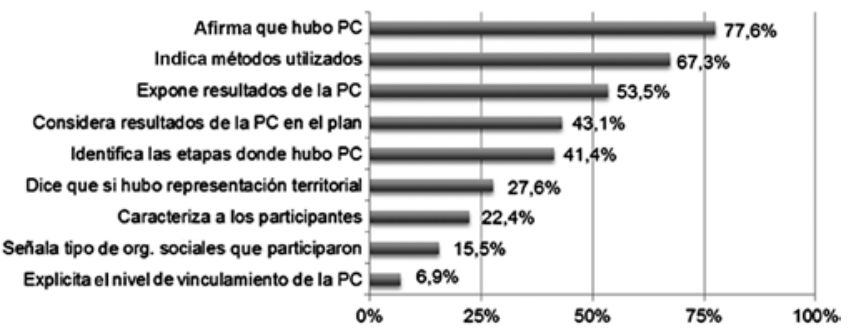

Fuente: elaboración propia.

Según estos datos y las distintas experiencias municipales analizadas, la participación ciudadana aparece como un desafío aún pendiente. Si bien se tiene clarísimo que el adjetivo "participativo" es algo que debe estar presente en todo PLADECO, no está claro cómo la participación puede ser aplicada. 
Según los entrevistados, existen ciertas limitantes que dificultan que este proceso se masifique. Entre éstas aparece la falta de una cultura participativa de la ciudadanía y el hecho de que, dado que el PLADECO no es vinculante, no existen estímulos concretos que inciten a que la comunidad se involucre. Sin embargo, la participación ciudadana también se ve como una gran oportunidad para legitimar acciones políticas y en particular para gatillar un proceso social en el cual se forje un vínculo entre la comunidad y el municipio, y entre los mismos vecinos quienes aprovechan la instancia para conocerse y organizarse. Así, las instancias participativas del PLADECO movilizan a que se avance hacia una comunidad más cohesionada y participativa. En este sentido, uno de los entrevistados expresa que "el PLADECO ha demostrado ser una herramienta de conversación ciudadana" (jefe de proyectos de planificación urbana, Viña del Mar)

\section{Rendición de cuentas}

A diferencia de lo que sucede con las funciones de planificación estratégica y participación ciudadana, la rendición de cuentas no es una característica de los PLADECO que esté definida como tal en la Ley $\mathrm{N}^{\circ}$ 18.695. Sin embargo, en la medida de que es un documento elaborado con su proceso participativo a su base, y a partir del cual se debe regir la planificación del accionar municipal, sumado a que se trata de información que figura en un documento de carácter público, se considera que el PLADECO tiene un importante potencial para facilitar el escrutinio público de la gestión municipal, más allá de las instancias de rendición de cuentas ya existentes a nivel de las comunas, tales como la cuenta pública anual. El análisis realizado a continuación apunta a saber si los documentos entregan claridad y transparencia respecto del nivel de compromiso del municipio frente a los objetivos y proyectos enunciados en el plan.

Para abordar la dimensión de rendición de cuentas, esta investigación analizará por separado las condiciones para la accesibilidad y comprensión acerca del potencial de rendición de cuentas propiamente tal, basado en los contenidos determinados que incorpore el PLADECO.

En primer lugar, de los índices calculados, el de accountability es el más bajo, siendo igual a 0.32, lo que quiere decir que en promedio los PLADECO cumplieron con sólo el 32\% de los contenidos exigidos en la pauta de evaluación en este aspecto. La mayor puntuación la obtuvo el documento de San Miguel $(0,86)$ y las más bajas corresponden a Villa Alemana, Renca, Recoleta, Quilpué, Quilicura, Providencia, Pudahuel, Nunnoa, Las Condes, La Serena, La Pintana, La Granja, El Bosque y Colina (0). El hecho de que casi un cuarto de los PLADECO estudiados (14 de 58) ni siquiera contemplen este ítem denota que la rendición de 
FIGURA 5. ACCOUNTABILITY.

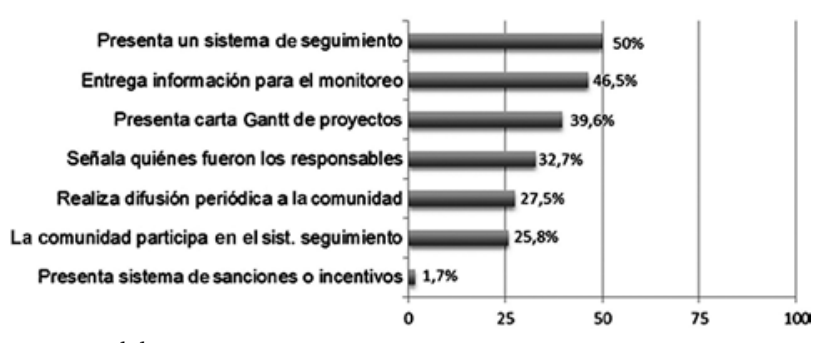

Fuente: elaboración propia.

cuentas es un ámbito que aún no ha sido instaurado en la lógica de este instrumento.

La figura 5 exhibe la cobertura que tuvieron ciertos ítems ligados a la rendición de cuentas, demostrando que la incorporación de un sistema de seguimiento, la entrega de información para el monitoreo ${ }^{51}$ y la presentación de una carta Gantt de proyectos fueron los ítems mejor cubiertos. Sin embargo, no son muchos los casos en los que el documento realiza una difusión periódica de los avances a la comunidad (27,5\%) y que éste permita que la comunidad participe en el seguimiento (sólo un $25,8 \%$ lo hace), por lo tanto se puede decir que en general el documento se remite a informar sobre los proyectos que pretende realizar (los cuales no necesariamente se llevan a cabo) pero no hace una rendición de cuentas del estado de avance, y de este modo no se utiliza como un instrumento

51 Se consideró cuando se informan iniciativas concretas con sus respectivos plazos de ejecución y las unidades responsables de éstas. activo sometido a evaluación periódica como establece la ley ${ }^{52}$.

Las experiencias estudiadas muestran que efectivamente hoy nos encontramos en un contexto en el que la ciudadanía es más exigente, pero aún no se ha instaurado una cultura que permita un monitoreo. Esto se da porque los municipios no han establecido mecanismos que lo permitan (lo único que existe en esta línea son los sistemas de reclamos), pero también se debe a la misma comunidad, ya que según uno de los entrevistados "la gente no tiene mucha cultura o mucha costumbre de por lo menos leer el PLADECO". Sin embargo, luego comenta que esto se debe a que "es un mamotreto imposible de leer, por lo que hay que hacer un esfuerzo para traducirlo en algo más o menos amable" (alcalde de Lo Prado). Se vuelve necesario entonces buscar la forma de hacer del PLADECO un instrumento activo y dinámico, ya que sólo así podrá cumplir con su función de rendir cuentas. "Una tarea que tenemos ahora en el nuevo PLADECO es establecer un sistema de seguimiento mucho más enfocado en los procesos (...) ojalá tener un software que te permita ir midiendo, eso sería fantástico" (asesora de gestión de proyectos SECPLA, Villa Alemana) ${ }^{53}$.

\footnotetext{
52 Ley $18.695,2006$.

53 Algo similar propone Valdivieso (2012).
} 


\section{FIGURA 6. ACCESIBILIDAD.}

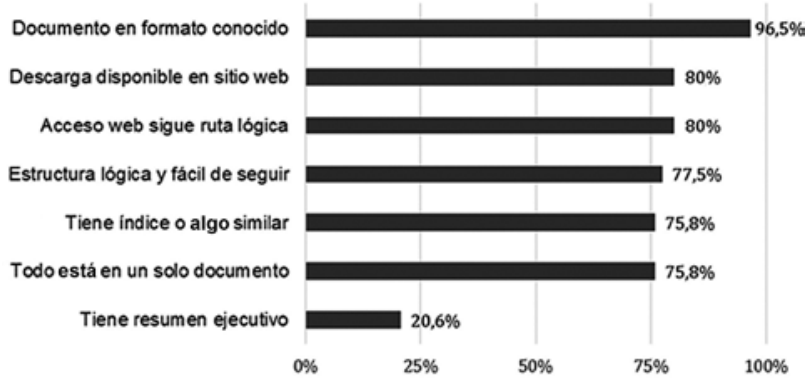

Fuente: elaboración propia.

De acuerdo al índice de accesibilidad y comprensión, es importante destacar que esta dimensión corresponde a aquella que es mejor cubierta por los 58 documentos analizados. En este aspecto, el índice obtenido de la muestra es el más alto entre todos los calculados, siendo igual a 0.71 , lo que quiere decir que en promedio los PLADECO cumplieron con el $71 \%$ de los contenidos exigidos en la pauta de evaluación. Las puntuaciones más altas las obtuvieron los documentos de Huechuraba y Lo Barnechea (1), mientras que la más baja corresponde a Quilpué, Tomé y El Bosque (0.29).

Tal como se muestra en la figura 6 , una proporción importante (cerca del 80\%) de los PLADECO analizados presenta buenas condiciones para su acceso y descarga desde la página web municipal, condición básica de accesibilidad. Respecto de los mismos documentos, éstos pueden tener ciertas características

196 revista invi 31(87) : 173-200, agosto de 2016 de formato que faciliten o dificulten la búsqueda de información y su comprensión. En esta línea, cerca del 75\% de los documentos presenta elementos mínimos que facilitan este trabajo, tales como la presencia de un índice y el diseño del PLADECO en un solo documento y no en varios, entre otros. No obstante, es esperable que cualquier documento elaborado por técnicos y profesionales cuente con condiciones mínimas de redacción y organización de sus contenidos que faciliten la búsqueda. Un aspecto negativo a destacar es que una fracción muy baja de los documentos presenta un resumen ejecutivo que permite una difusión más rápida y sintética de sus contenidos más importantes (20.6\%).

Una lectura algo más perspicaz sobre este resultado nos lleva a reflexionar acerca de por qué el ítem acceso y comprensibilidad fue mejor evaluado que los otros. Las dimensiones mejor cubiertas de éste corresponden a características formales que visibilizan y constatan públicamente la existencia del documento, y en esta medida entregan -sin mayor esfuerzoa las autoridades y su escrutinio una evidencia de cumplimiento con la ley. En este sentido destaca el alto porcentaje de documentos disponibles efectivamente en el sitio web del municipio (80\%), así como que un alto porcentaje de los PLADECO revisados en este estudio cumple con otros de los mínimos establecidos por ley: ser un instrumento de planificación vigente y uno que constata la realización de un proceso de participación ciudadana. 
Más allá de estar vigente y de su publicación en la web $^{54}$, no se ha invertido la misma energía en socializar el documento con la comunidad por otros medios. En palabras del asesor de la SECPLA de Coronel: "Yo diría que al PLADECO lo conocen las personas que están en la junta de vecinos, en organizaciones territoriales, fundamentalmente". En esta línea, desde los mismos municipios diagnostican que el documento está poco socializado en parte por ser poco amable en su lenguaje para el ciudadano. "No está en un lenguaje apto. No entienden muchos conceptos técnicos. Por eso tú tienes que adaptarlo también a la realidad del vecino" (SECPLA, Lo Prado). De este modo, nuevamente adquiere relevancia el bajo porcentaje de documentos que cuentan con un resumen ejecutivo.

\section{Conclusiones}

El PLADECO como instrumento rector de planificación y gestión comunal debe ser capaz de expresar la visión y los anhelos de la comunidad para orientar a las autoridades municipales. No obstante, el resultado de este trabajo refleja una situación bastante desalentadora, ya que en la mayoría de las comunas el cumplimiento de los compromisos establecidos en el desarrollo del PLADECO resulta ser inferior al 50\% en todos los ejes de análisis, a excepción de accesibilidad.

Las razones que explican este resultado responden al hecho de que los municipios se encargan de asegurar el cumplimiento mínimo establecido por la ley (que es contar con un PLADECO vigente), pero rindiendo cuentas al fiscalizador oficial en términos generales, pero no en específico a la ciudadanía. Así, el hecho de que aspectos relativos a la participación y accountability evidencien bajas exigencias de cumplimiento de los PLADECO responde a que la ley sólo exige que haya habido un proceso participativo sin precisar modo ni incidencia, y no plantea nada acerca de accountability.

Junto al análisis de los 58 PLADECO que han sido parte de la metodología utilizada, se han efectuado entrevistas a actores claves del mundo municipal, las cuales si bien conciben varias limitaciones del instrumento, es muy probable que el juicio desde la comunidad y de agentes externos sea aún más crítico respecto a la incidencia del PLADECO como instrumento rector de la planificación y gestión municipal. A partir de la bibliografía revisada se confirma en gran medida el juicio anterior.

Adicionalmente, los resultados de este trabajo permiten establecer que no existe ninguna correlación

54 Medio de comunicación que excluye a una fracción de la población que no maneja las herramientas computacionales básicas para acceder a esta información. 
significativa que haga pensar que a mayores recursos disponibles o calidad de vida de una comuna, se obtengan PLADECO con mayores niveles de cumplimiento de acuerdo a las dimensiones planteadas. Las entrevistas identificaron que uno de los principales factores que permiten que el PLADECO sirva como instrumento rector de la planificación y gestión municipal es la voluntad política de las autoridades de turno. Esto se debe principalmente a que en el marco de una normativa poco exigente, aquellos casos que buscan "hacer más que el mínimo" logran destacar. Esto se da especialmente cuando el alcalde lidera de buena forma este proceso.

En materia de planificación los PLADECO quedan al debe, ya que en la mayoría de los documentos revisados éstos se limitan a señalar qué quieren alcanzar proponiendo objetivos e iniciativas en forma genérica, sin embargo, no se elaboran estrategias concretas sobre cómo lograr tales objetivos. También queda en evidencia que los PLADECO tienen una alta dependencia financiera, lo que junto con las contingencias sociales, ambientales y políticas, dificulta la planificación urbana de los gobiernos locales. Por eso, se vuelve evidente la necesidad que tienen los municipios en Chile de contar con una mayor autonomía financiera, así como dotarse con personal calificado capaz de desarrollar estrategias para llevar a cabo los objetivos planteados.

198 revista invi 31(87) : 173-200, agosto de 2016
Finalmente, se considera que la metodología aportada por este trabajo puede aplicarse a futuros esfuerzos de investigación en este campo de estudio, particularmente en el propósito de profundizar sobre características que determinados tipos de PLADECO tienen y que garantizan su mayor cumplimiento, así como en el objetivo de emprender modificaciones de orden técnico y legal para que este instrumento sea realmente un instrumento rector de la gestión municipal en Chile.

\section{Bibliografía}

ARNSTEIN, Sherry. A ladder of citizen participation. Journal of the American Institute of Planners. 35(4): 216-224, julio 1969. [Fecha de consulta: mayo 2015]. ISSN 0002-8991. DOI 10.1080/01944366908977225.

BAÑo, Rodrigo. Participación ciudadana: elementos conceptuales. En: CORREA, Enrique, ed. y NOÉ, Marcela, ed. Nociones de una ciudadanía que crece. Santiago, Chile, FLACSO-Chile. 1998. P. 1537. Serie Libros FLACSO. ISBN 956-205-126-9.

BRENNER, Neil. Tesis sobre la urbanización planetaria. [En línea]. Nueva Sociedad. (243): 38-66, enero 2013. [Fecha de consulta: mayo 2015]. ISSN 0251-3552. Disponible en: http://nuso.org/media/ articles/downloads/3915_1.pdf.

CAVIGLIA, Franco. Municipio, participación y desarrollo: una problemática del siglo XXI. Buenos Aires, Pazcuaro. 1998. 183 p. ISBN 9879720008. 
CRESPO, José Antonio. Fundamentos políticos de la rendición de cuentas. México, Auditoría Superior de la Federación. 2001. 58 p. Serie cultura de la rendición de cuentas. ISBN 968-5500-00-2.

DE LA MAZA, Francisca; CAMPOS, María Sylvia; VEGA, Patricia y GAETE, Tomás. Propuesta para incorporar a participación intercultural en los planes de desarrollo comunal de la región de la Araucanía. En: CAMINO al Bicentenario: propuestas para Chile. Santiago, Pontificia Universidad Católica de Chile. 2008. P. 155-191. ISBN 978-956-14-1035-0.

ESPINOZA, Claudia. La territorialización de la participación ciudadana en el marco de la elaboración de planes de desarrollo comunales. [En línea]. Tiempo y Espacio. (25), 2010. [Fecha de consulta: mayo 2014]. ISSN 0719-0867. Disponible en http://revistas.ubiobio.cl/index.php/TYE/article/ view/1773/1717

FERNÁNDEZ, María Ignacia, coord. y JORDAN, Ricardo, coord. Manual de elaboración del Plan de Desarrollo Comunal (PLADECO). Santiago, Chile, Cepal, SUBDERE. 2009. 118 p. ISBN 978-956-8468-14-9.

FUENTES, Luis y ORELLANA, Arturo. Gobernabilidad metropolitana en Bogotá y Santiago: institucionalidad divergente, resultados convergentes. Ciudad y territorio: Estudios territoriales. 15(175): 213-219, 2013. [Fecha de consulta: septiembre 2015]. ISSN 1133-4762.

GIMÉNEZ, Pilar, ed. y UGARTE, José Ramón, ed. Política nacional de desarrollo urbano: ciudades sustentables y calidad de vida. PNUD, MINVU. 2014. ISBN 978-956-9432-05-7.

JORQUERA, Álvaro y PINEDA, Héctor. El plan de desarrollo comunal como una oportunidad para la participación ciudadana: creación de observatorio de desarrollo local para la comuna de San Bernardo, Chile. Revista de Estudios Políticos y Estratégicos. 2(1): 66-89, 2014. ISSN 0719-3688.

LEY 18.695. Fija el texto refundido, coordinado y sistematizado de la ley $\mathrm{n}^{\circ} 18.695$, orgánica constitucional de municipalidades. [En línea]. Ley Chile. 2006. Disponible en: http://bcn.cl/luuyl.

MIDEPLAN. Aportes a la descentralización: sistema nacional de planificación y gestión en el territorio. Santiago, Chile. 2006. 66 p.

MONTECINOS, Egon. Descentralización y democracia en Chile: análisis sobre la participación ciudadana en el presupuesto participativo y el plan de desarrollo comunal. Revista de Ciencia Política. 26(2): 191-208, 2006. ISSN 0718-090X. DOI 10.4067/ S0718-090X2006000200011.

SAN MARTíN, Héctor. Accountability y transparencia en la gestión pública: una propuesta. [En línea]. Horizontes Empresariales. 7(1): 71-82, mayo 2008. [Fecha de consulta: abril 2014]. ISSN 0719-0875. Disponible en: http://revistas.ubiobio.cl/index. php/HHEE/article/view/2049.

SÁNCHEZ, Fernando. Planificación estratégica y gestión pública por objetivos. Santiago de Chile, ILPES-CEPAL. 2003. 78 p. Serie gestión pública. ISBN 9213221517. 
SANTANA, Gabriel. Instrumentos de planificación de la gestión municipal chilena. Valdivia, Universidad Austral de Chile. 2012. 116 p.

THAYER, Luis Eduardo y ELIZALDE, Antonio. La dimensión de lo público. Sociedad civil y Estado. Polis. 10(30): 7-15, diciembre 2011. ISSN 0718-6568. DOI 10.4067/S0718-65682011000300001.

VALDIVIESO, Gonzalo. ¿Para qué sirve el PLADECO? [En línea]. PLANEO. (7), septiembre 2012. [Fecha de consulta: abril 2014]. Disponible en: http://revistaplaneo.uc.cl/planeo-hoy/columnas/lugares/ para-que-sirve-el-pladeco.

VELÁSQUEZ, Fabio y GONZÁLEZ, Esperanza. ¿Qué ha pasado con la participación ciudadana en Colombia? Bogotá, Fundación CORONA. 2003. 456 p. ISBN: 958-97199-7-X. 